\title{
Consumer perspectives in surgical research and audit
}

\author{
Eleanor Ahern, Prema Thavaneswaran \\ ASERNIP-S, Royal Australasian College of Surgeons \\ Wendy Babidge, Guy J. Maddern \\ ASERNIP-S, Royal Australasian College of Surgeons and University of Adelaide and The \\ Queen Elizabeth Hospital
}

Objectives: The aim was to learn about perspectives of consumers contributing to the work of the Division of Research, Audit and Academic Surgery of the Royal Australasian College of Surgeons. The research arm of the Division has worked with consumers since it was formed in 1998.

Methods: Nine consumers who worked with the Division over the past 5 years completed (1) a written survey focused on their background and past experience, and (2) a semi-structured phone interview focused on their motivations for becoming involved in this work; their role; the evolution of the role of consumers in healthcare research; and what health information for consumers should contain.

Results: Participants came from various backgrounds and had different motivations for being involved. A common theme was concern about uncertainties in surgery and the need to provide consumers with information about potential benefits and risks of a procedure. Participants believed that a consumer presence was vital in research on surgical procedures, and that the content and wording of consumer information must be chosen carefully in order for the public to use it in a meaningful way. They also acknowledged the changing role of the consumer, who was rapidly becoming a partner in the doctor-patient relationship.

Conclusions: In surgical research and audit, the consumer perspective is unique and informed by a wealth of experience. The findings of this study may be of interest to other health technology assessment and associated agencies seeking to involve consumers within their own research process.

Keywords: Consumer participation, Clinical audit, Surgical procedures, Operative, Evidence-based medicine

Worldwide, consumer input into health services research has become increasingly important in recent years (2). Consumer

\footnotetext{
The authors acknowledge the consumers involved with the Division of Research, Audit and Academic Surgery (RAAS) of the Royal Australasian College of Surgeons (the College of Surgeons) for giving their time to discuss their involvement in the Division's activities. The authors also thank Ms Assunta Morrone from the Health Issues Centre, La Trobe University, Victoria for her assistance with consumer consultation methods. The Australian Safety and Efficacy Register of New Interventional Procedures-Surgical (ASERNIP-S) project is part of the RAAS Division. This work has been funded by the South Australian Department of Health.
}

involvement in this area relates to an active relationship between consumers, researchers, and healthcare providers (2). It has been suggested that involving consumers may improve the quality of research and increase its relevance (2). Many research organizations value the unique perspective of consumers, who can explain the impact of a disease and its treatment, which may involve the use of a healthcare technology $(5 ; 7)$. In 2005 , the results of a survey of the International Network of Agencies for Health Technology assessment (INAHTA) showed that most members involved consumers 
in some aspects of their programs, although not always routinely, and that this involvement was likely to increase in the future (6). In Australia, peak consumer organizations like the Breast Cancer Network Australia (BCNA) have recommended that consumer input be included in all aspects of research (3). Additionally, in a recent review of health technology assessment (HTA) in Australia, the Consumers' Health Forum of Australia (CHF) recommended that "consumer input be a core component of the HTA system in Australia to ensure that the system meets consumer needs and recognizes the impact of health technologies on their lives" (1).

Traditionally patients relied upon the advice of their doctor, who in turn depended on information gained through the medical history and examination of the patient to make their diagnosis; this patient-doctor partnership continues today (4). HTA should be used to inform this shared decisionmaking process, allowing the patient and doctor to make informed decisions together (4). These assessments, however, are often made on groups of patients in a clinical setting, such as through randomized controlled trials, which aim to be generalizable rather than taking into account individual patient preferences (4). Some methodologies support what some perceive as a doctrine of paternalism, providing formulas for doctors to calculate what is good for patients (4). These drawbacks highlight the need to involve consumers in the HTA process from an early stage, so that the evidence produced can be a useful tool in decision making for both doctor and patient. This is particularly challenging in the area of surgery, one of the most complex and rapidly evolving areas of medicine.

Consumers are increasingly involved at many different levels in surgical research, which may include the assessment of new and emerging surgical techniques and technologies (health technology assessment) as well as the establishment of surgical audits. An example of this is the involvement of consumers in the work conducted by the Division of Research, Audit and Academic Surgery (RAAS) of the Royal Australasian College of Surgeons (the College of Surgeons).

\section{HOW RAAS INVOLVES CONSUMERS}

The Australian Safety and Efficacy Register of New Interventional Procedures-Surgical (ASERNIP-S), which is part of the Division, has worked with consumers since the organization was formed in 1998 to assess the safety and efficacy of new surgical procedures. Since its inception, ASERNIP-S has had at least one consumer representative on its management/advisory committee; the consumer representatives comment on systematic reviews, and work with groups of surgeons and researchers to prepare consumer information. ASERNIP-S establishes links with national consumer organizations, including the CHF, of which the College of Surgeons became an organizational member in 2008. ASERNIP-S has also been an active member of the Interest Sub-Group of Health Technology Assessment International (HTAi) on pa-

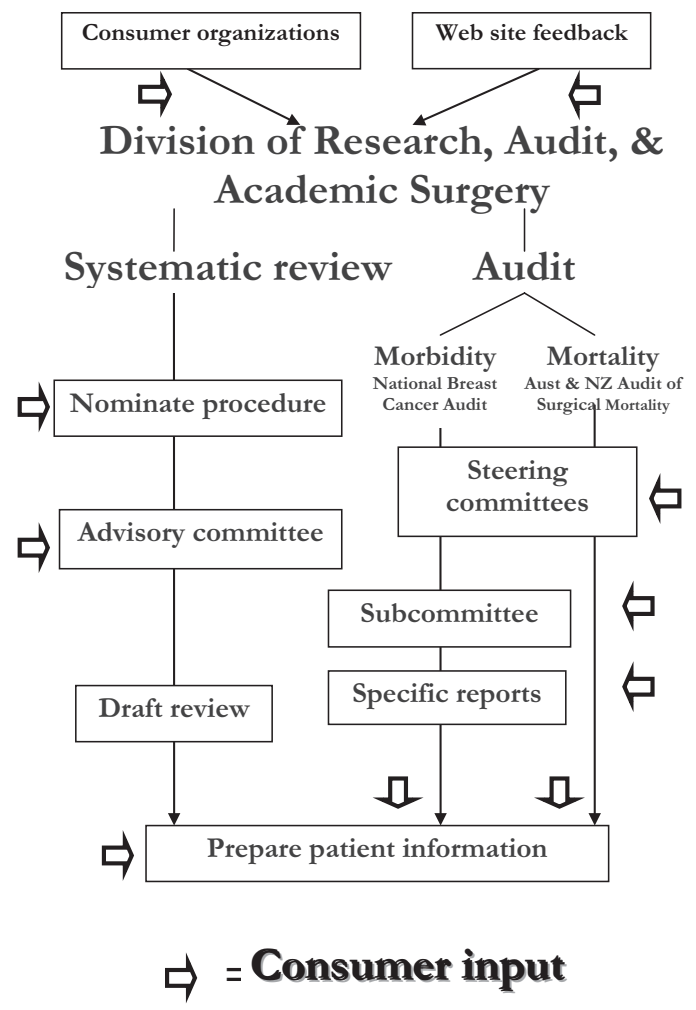

Figure 1. Consumer input into surgical research and audit in the Division of Research, Audit and Academic Surgery.

tient and citizen involvement in health technology assessment since 2006, and provided input on the HTAi consumer and patient glossary (8). Consumers are also involved in audits conducted by the Division, namely the National Breast Cancer Audit and the Australian and New Zealand Audit of Surgical Mortality. Through the ASERNIP-S Web site, consumers can nominate surgical procedures for assessment and access all reports prepared by the Division. The many ways that consumers provide input into the Division is shown is Figure 1. More detailed information on how RAAS involves consumers is provided in Supplementary Table 1, which can be viewed online at www.journals.cambridge.org/thc2011025.

\section{METHODS}

\section{Participants}

Nine consumers who worked with the RAAS Division over the past 5 years were identified. All prospective participants were initially contacted by means of email, and the purpose and scope of the study explained. These participants comprised consumer representatives working with the Division, and members of a peak consumer organization that collaborated with the Division on a specific project. In this study, a "consumer" may be a surgical patient, carer, member of the public or a member of an organization. A "consumer representative" puts forward views on behalf of consumers of health care. 
Table 1. Questions in the Written Survey and Semi-structured Telephone Interview

\section{Written survey}

1. How long have you been a consumer representative or working with a consumer organisation?

2. How long have you worked with the RAAS Division?

3. What other organizations have you been involved with as a consumer?

4. Do you come from a health background?

5. Has your previous experience helped you in your work here?

6 . What training have you had for your role as a consumer?

Semi-structured telephone interview

1 . What is your motivation for being involved?

2. How do you see your role in the RAAS Division?

3 . How has your role evolved?

4. How has the role of consumers in general changed?

5. Would you like your role to develop in the RAAS Division? If

so, and the necessary resources were available, how?

6. What should the ideal consumer information contain?

\section{Data Collection}

A survey form was sent by email to the participants, who all responded. All participants were contacted to confirm a suitable date and time for the semi-structured telephone interview. These were conducted by telephone with individual participants. This format was used so that participants would feel free to discuss issues which were important to them. Each interview was conducted by one (E.A.) or two (E.A. and P.T.) ASERNIP-S researchers. At the start of each interview, each participant was asked if the proceedings could be recorded and all consented. The researchers explained that the responses of all participants would be de-identified. All interviews were recorded and later transcribed by the researcher(s) who conducted the interviews.

\section{Interview Questions}

The questions in the survey were focused on the backgrounds of the participants. The questions used to guide the discussion during the semi-structured telephone interviews focused on the consumers' motivations for becoming involved in this work; their role; the evolution of the role of consumers in healthcare research; and, in relation to health information prepared for consumers, ideally what that consumer information should contain. Participants were encouraged to talk about any aspect of these topics. The questions used in the written survey and semi-structured phone interviews are listed in Table 1.

\section{Data Analysis}

Responses to all questions were de-identified following transcription. Two authors (E.A. and P.T.) separately identified themes emerging from the interviews; following discussion, both authors agreed upon the most commonly reported themes that were relevant to the aim of the study. The two authors then grouped the responses of individual participants
Table 2. Participant Responses to the Written Survey

Length of time working as a consumer representative or with a consumer organization

Median 10 years (range 3-33 years)

Length of time working with RAAS Division $1-7$ years

Involvement with other consumer organizations

Consumers Health Forum (2/9)

Consumer groups for specific medical condition (5/9)

Health councils (4/9)

Government committees (3/9)

Cochrane Collaboration (3/9)

Hospitals/health centers (4/9)

None (1/9)

Background (health or other)

Health (4/9)

Teacher/academic (4/9)

Priest (1/9)

Journalist (1/9)

Pharmacologist (1/9)

Audio engineer (1/9)

Lawyer (1/9)

Experience with health issues, both as an individual and through family and friends (1/9)

How does previous experience help?

Preparing consumer information that is readable and relevant

Understanding different perspectives of consumers

Differences in uptake of an emerging surgery between countries

Working with people from different backgrounds on committees

Policy and legislation

Systematic reviews

Community expectations, ethical standards and medical systems

Not sure of the question

Training

Consumers Health Forum (5/9)

Parish and hospital (1/9)

Talking with patients (1/9)

Reading journals (1/9)

Own organization in-house (2/9)

None (1/9)

into these themes, and described them narratively. Direct quotes were used wherever possible.

\section{RESULTS}

All nine consumers who were invited to participate in the study completed the survey form and took part in the semistructured telephone interview (100 percent participation rate).

\section{Written Survey}

The responses of the participants to the written survey have been grouped together and summarized in Table 2. Most participants had provided consumer input to at least one organization for an average of more than 12 years. Four consumers worked with the research arm of the RAAS Division and five with the audit arm. Many of the participants were also 
involved with several other consumer organizations. Consumers came from a variety of backgrounds, and their previous experience had helped in many ways. All but one of the consumers had received some training. One participant from a consumer organization which supported consumers suffering from a particular health condition said that working and listening to consumers had provided a deep perspective of the challenges faced by them, and had helped the participant advocate on their behalf.

\section{Semi-structured Telephone Interview}

The themes which emerged from the responses of participants during the semi-structured telephone interview are summarized below. A selection of quotes taken from the responses appears in Supplementary Table 2, which can be viewed online at www.journals.cambridge.org/thc2011025.

Consumers wanted to be involved with the organization for many reasons, including to inform patients of benefits and harms of surgery, to improve the quality of care, to use professional skills to translate difficult information for patients, to improve consumer confidence in treatment management, for family reasons, it was part of their role at work, to help the organization set up relationships with consumer groups, to represent the community, and because of the challenge.

When participants were asked how they perceive their role in the RAAS Division, one was unsure due to recent changes in the type of work undertaken by that project, while other consumers said it was their role to bring the patient perspective to the research process, remind of the uncertainty in treatments, aid communication between stakeholders, represent the ordinary person on the street, translate information for other consumers, and work toward a shared purpose.

One participant was happy that the participant's role in the RAAS Division had not changed; however, most consumers thought their role had evolved in some way, as they became more familiar with the committees, from being a member of a committee to driving decisions, through partnerships the RAAS Division made with consumer organizations, and as the audit grew from one region to a national project.

Participants thought the role of consumers in general had changed so that there is more of a partnership between patient and doctor; patients use health information to help them make decisions; consumers are more demanding and have higher expectations of outcomes; consumer representation has increased; consumers actively seek information from a wide variety of sources, including the Internet; and consumers can fund and drive projects.

Seven of the nine participants thought their role in the RAAS Division could be developed, if resources were available. These changes included the following: more evaluative work and consumer feedback, increased consumer representation on committees, more involvement with the RAAS Division, increased understanding of the way the committee works, and meeting to clarify roles and the direction of the RAAS Division. However, one consumer did not see the need to develop the role, and another did not answer as there had been uncertainty regarding the continuation of that project.

Most consumers thought that the language used in consumer information should be plain, avoiding jargon and acronyms, and should not contain words that may alarm patients. The participants thought that ideally consumer information would be written in appropriate language and tone, contain adequate content on benefits and risks, spell out the level of evidence, clearly state sources of funding, be tailored to the individual who is using it, contain definitive statements on findings, be handed over during discussions with the doctor, contain local details (e.g., government reimbursements), contain contact details for further information, and be available in different media.

\section{DISCUSSION}

Since the formation of ASERNIP-S under the umbrella of the College of Surgeons in 1998, when one consumer gave advice on systematic reviews and consumer summaries, the involvement of consumers in the organization has grown considerably. Since that time, two consumer representatives have worked on the organization's Advisory Committee to help develop the content, presentation and dissemination of more than 45 consumer summaries, thus ensuring that the information available to patients and the public is readable and relevant to their needs. The public can provide feedback on consumer summaries or receive advice on procedures through the Web site at www.surgeons.org/asernip-s. In addition, consumer representatives have helped the organization to strengthen links with peak consumer groups in Australia.

Consumers have also become increasingly involved in the audit activities of the RAAS Division of the College of Surgeons. Consumers working on audit activities through committees found their roles had evolved, in some cases to the point of driving and funding research projects. The number of individuals providing a consumer voice on committees had also increased. Recently, consumers have worked collaboratively to prepare consumer summaries on audit reports so that consumers can access and understand the latest data more easily.

This study attempted to gain an understanding of the perspective of the consumers who provide input into the research and audit processes of the RAAS Division. Participants came from a variety of backgrounds and had different motivations for being involved. A common theme was concern about the uncertainties in surgery and the need to provide consumers with information about the potential benefits and risks of a procedure. Participants believed that a consumer presence was vital in research conducted into surgical procedures, and that their previous experience, both professional and personal, equipped them to put forward the views and concerns of patients and the general public. Key findings from consultations with health consumers in the HTA review highlight the need for "transparent mechanisms for genuine 
involvement of consumers at all stages" of the HTA process, and "consumer representation in all assessment and review processes" (1). The Division has involved consumers at many different levels of the process, as seen in Figure 1.

The participants in this study acknowledged the changing role of the consumer of health care, who was rapidly becoming a partner in the doctor-patient relationship. Patients were now seeking more information on their treatments, from a variety of sources, and had greater expectations of positive outcomes. In some cases, the participants' own roles providing input on research committees evolved as they became more vocal and were given more opportunities. Others still felt isolated and needed more discussion on what their contribution to the research process could be. One participant in the survey expressed uncertainty regarding the participant's role, due to a reduction in the number of committee meetings. This had occurred as a result of changes in funding arrangements as the project evolved.

Participants wanted to let consumers know about the types of research or audits being conducted, and the outcomes of these activities. They also believed that the content and wording of consumer information needed to be chosen carefully for the public to use it in a meaningful way.

A limitation of the study was the small number of participants, who had all worked recently (within the last five years) with the RAAS Division. The study may also have benefited from external evaluation, as two researchers in the organization conducted and transcribed the interviews, which may have introduced bias into the interpretation of participant responses. This was an Australian organization, so the findings are applicable to the Australian healthcare context and may not translate to organizations working in healthcare systems in other parts of the world.

The role of the consumer in the HTA process will continue to evolve. Over time the RAAS Division has sought and benefited from the input of consumers at an increasing number of levels. This input has grown in both number and depth. Consumers bring a range of experience to these roles, and are drawn to the work for many different reasons.

\section{LESSONS LEARNED}

By learning about the perspectives of these consumers, the organization hoped to gain a greater understanding of how consumers may be involved in different ways in the future. Regarding study design, the survey questions could have been refined to ensure that the meaning was clear to all study participants. Another improvement could have been to have worked with an independent researcher, who was not involved in conducting the interviews, to collate the data collected. In the responses of the participants to the survey questions, the importance of providing consumers with appropriately worded information about the potential benefits and risks of a procedure was highlighted, as was the need for a consumer presence in research into surgical procedures.
Other lessons learned by the organization were that the role of consumers evolves as they are given more opportunities. Isolation of consumer representatives and lack of sufficient information about their role are issues which need to be addressed. Another important issue is the training consumers need to support them in their role in research organizations, and who will provide that training. One of the eight principles of successful consumer involvement in NHS research provided by Telford in 2004 is to offer training to consumers to enable them to be involved in research (9). Although the Division provides mentors to support consumers, more formal training could be of benefit in the future. The Division currently meets the other seven indicators.

Through this study, the organization has gained a greater understanding of consumer involvement in surgical research and audit, and hopes to use this knowledge to strengthen consumer participation in the future. The issues raised in this study could be considered by health technology assessment and associated agencies seeking to involve consumers within their research process.

\section{SUPPLEMENTARY MATERIAL}

Supplementary Table 1

Supplementary Table 2

www.journals.cambridge.org/thc2011025

\section{CONTACT INFORMATION}

Eleanor Ahern, BA, MA, ASERNIP-S (Eleanor.Ahern@ surgeons.org), Royal Australasian College of Surgeons, 199 Ward Street, North Adelaide, South Australia 5006, Australia Prema Thavaneswaran, BSc (Hons), PhD (Prema. Thavaneswaran@surgeons.org), ASERNIP-S, Royal Australasian College of Surgeons, 199 Ward Street, North Adelaide, South Australia 5006, Australia

Wendy Babidge, B App Sci (Hons), PhD, Grad Dip Bus (wendy.babidge@ surgeons.org), Guy J. Maddern, MBBS, MS, PhD, MD, FRACS (guy.maddern@adelaide.edu.au), ASERNIP-S, Royal Australasian College of Surgeons, 199 Ward Street, North Adelaide, South Australia 5006, Australia; Department of Surgery, University of Adelaide and The Queen Elizabeth Hospital, Adelaide, South Australia, Australia

\section{CONFLICT OF INTEREST}

The authors' institution received funding from the South Australian Department of Health for this study.

\section{REFERENCES}

1. Australian Government Department of Health and Ageing. Review of Health Technology Assessment in Australia; 2009. http://www.health.gov.au/internet/main/publishing.nsf/Content/ 00E847C9D69395B9CA25768F007F589A/\$File/hta-reviewreport.pdf (accessed December 13, 2010). 
Ahern et al.

2. Boote J, Telford R, Cooper C. Consumer involvement in health research: A review and research agenda. Health Policy. 2002;61:213-236.

3. Breast Cancer Network Australia. Summary of recommendations from 2004 Conference. http://www.bcna.org.au/ index 2.php?option=com_content\&task=view\&id=390\&pop $=1$ (accessed February 3, 2010).

4. Bridges J, Jones C. Patient-based health technology assessment: A vision of the future. Int J Technol Assess Health Care. 2007;23:30-35.

5. Facey K, Boivin A, Gracia J, et al. Patients' perspectives in health technology assessment: A route to robust evidence and fair deliberation. Int J Technol Assess Health Care. 2010;26:334-340.
6. Hailey D, Nordwall M. Survey of the involvement of consumers in health technology assessment programs. Int J Technol Assess Health Care. 2006;22:497-499.

7. Health Equality Europe. Understanding health technology assessment; 2009. http://www.htai.org/index.php? $\mathrm{id}=85$ (accessed February 3, 2010).

8. The Health Technology Assessment International (HTAi) Interest Sub-Group for Patient and Citizen Involvement in HTA. HTA $i$ consumer and patient glossary: a beginner's guide to the words used in health technology assessment; $2009 \mathrm{http}: / /$ www.htai.org/index.php?id=85 (accessed February 3, 2010).

9. Telford R, Boote JD, Cooper CL. What does it mean to involve consumers successfully in NHS research? A consensus study. Health Expect. 2004;7:209-220. 